\title{
A lay perspective on prioritization for intensive care in pandemic times: Vaccination status matters
}

Philipp Sprengholz ${ }^{1,2 *}$, Lars Korn ${ }^{3}$, Lisa Felgendreff ${ }^{2}$, Sarah Eitze ${ }^{2}$, and Cornelia Betsch ${ }^{2,3}$

${ }^{1}$ University of Jena, Jena, Germany

${ }^{2}$ University of Erfurt, Erfurt, Germany

${ }^{3}$ Bernhard Nocht Institute for Tropical Medicine, Hamburg, Germany

*Corresponding author: Philipp Sprengholz, philipp.sprengholz@uni-erfurt.de 


\begin{abstract}
During a pandemic, demand for intensive care often exceeds availability. Experts agree that allocation should maximize benefits and must not be based on whether patients could have taken preventive measures. However, intensive care units (ICUs) are often overburdened by individuals with severe COVID-19 who have chosen not to be vaccinated to prevent the disease. This article reports an experiment that investigated the German public's prioritization preferences during the fourth wave of the coronavirus pandemic $(N=1,014)$. In a series of scenarios, participants were asked to decide on ICU admission for patients who differed in terms of health condition, expected treatment benefits, and vaccination status. The results reveal an ingroup bias, as vaccinated individuals preferred to allocate more resources to the vaccinated than to the unvaccinated. Participants also favored admitting a heart attack patient rather than a COVID-19 patient with the same likelihood of benefiting from ICU admission, indicating a preference for maintaining regular ICU services rather than treating those with severe COVID-19. Finally, participants were more likely to admit a patient to intensive care when this meant withholding rather than withdrawing care from another patient. The results indicate that lay prioritizations violate established allocation principles, presaging potential conflicts between those in need of intensive care and those who provide and allocate it. It is therefore recommended that allocation principles should be explained to enhance public understanding. Additionally, vaccination rates should be increased to relieve ICUs and reduce the need for such triage decisions.
\end{abstract}




\section{Introduction}

When COVID-19 began to spread in 2020, intensive care units (ICUs) filled rapidly, and there were images of crowded hospitals across the world turning away severely infected patients (Nacoti et al., 2020). The approval and rollout of vaccines was perceived as a turning point. As vaccination reduces the chances of critical infection and transmission of the disease (Tenforde et al., 2021), experts called on governments to vaccinate at least $85 \%$ of the population (Burki, 2021; del Rio, Malani, \& Omer, 2021). To date, vaccination rates in most countries have failed to reach this target; for instance, despite extensive efforts to ease, promote, and incentivize vaccination, rates in the US were below 60\% in November 2020 (Our World in Data, 2021). Similarly, vaccination rates in Germany have settled at around $70 \%$, which was not enough to prevent a fourth drastic surge of infections and deaths (Wichmann et al., 2021). As ICUs filled again and hospitals had to turn away patients with severe conditions like intracerebral hemorrhage (Tagesschau, 2021), discussion turned to the allocation of intensive care beds. Should unvaccinated patients with severe COVID-19 be deprioritized for admission when there are too few ICU beds for all of those in need?

As provision of intensive care requires significant resources, ICU capacity is necessarily limited, and ICU beds are generally reserved for patients whose recovery depends on this high level of care. Typically, patients who are expected to recover outside ICU and those who are expected to die regardless of medical intervention are not admitted (Vincent \& Creteur, 2020). However, despite this emphasis on specific prognoses, ICU capacity may be insufficient from time to time, and when demand for intensive care exceeds the available capacity, triage is required. Persad, Wertheimer, and Emanuel (2009) identified four basic principles for allocating scarce medical resources.

(1) Maximizing benefits (utilitarianism): According to this principle, intensive care should be allocated to those who are most likely to benefit. Maximizing benefits can be understood as saving the greatest number of individual lives or preserving the most life years by prioritizing those who are expected to survive longest and with reasonable quality of life following treatment. Clearly, this latter decision depends on information about patient age as well as prior medical conditions and comorbidities.

(2) Favoring the worst off (prioritarianism): This principle favors allocation of scarce resources to patients with the worst prospects if left untreated, disregarding posttreatment prognoses. While this typically means prioritizing the sickest, it can also mean 
favoring younger patients, who are considered to be in greater need because they will potentially lose more life years.

(3) Treating people equally (egalitarianism): On this principle, all patients should have equal access to intensive care. This can be achieved by implementing a first-come first-served rule or a lottery system that randomly assigns ICU capacity in conditions of scarcity.

(4) Promoting and rewarding instrumental value (instrumentalism): This principle prioritizes certain patients in order to maintain critical infrastructures. For instance, healthcare workers may be privileged because they may save others in the future.

As no single principle can adequately incorporate all of the morally relevant considerations, allocation systems typically invoke multiple principles. However, approaches to selecting, weighting, and operationalizing principles vary across interventions (see for example, Christian et al., 2006; Persad, Peek, \& Emanuel, 2020). In the case of ICU admission, scholars agree that the most important concern is to maximize benefits (Emanuel et al., 2020; Marckmann et al., 2020; Vincent \& Creteur, 2020). Given the constraints on time and information during a pandemic, it is reasonable to maximize the number of patients likely to survive treatment with a reasonable life expectancy by prioritizing younger individuals with fewer coexisting conditions for admission to intensive care. Similarly, it seems reasonable to prioritize healthcare professionals and other key workers over other occupational groups (Emanuel et al., 2020). Patients with similar prognoses (and similar instrumental value) should be treated equally by implementing random allocation. In other words, triage decisions should not be influenced by a patient's wealth or social status or by any personal relationship with medical staff (Vincent \& Creteur, 2020).

As triage systems do not typically take account of whether patients are to blame for their condition, allocation of intensive care should be no different for patients with COVID19 and those with other severe conditions like heart failure, stroke, or cancer (Emanuel et al., 2020). Although there would have been significantly fewer hospitalizations during the COVID-19 pandemic if everyone was vaccinated against the disease, unvaccinated patients with severe COVID-19 are nevertheless entitled to intensive care. Imagine, for example, that two comparable persons are competing for an ICU bed: one with COVID-19 and one who has suffered a heart attack. According to the above principles, there should be no clear preference, as both are equally sick and would benefit to a comparable extent from intensive 
care. In this case, random selection is justified. However, some laypeople might disagree with this recommendation. In countries where vaccination against COVID-19 is readily accessible and available to everyone, high incidence of severe infection and the need for triage could easily be avoided. Previous research has shown that people are blamed for their own health problems when perceived as irresponsible (Decety, Echols, \& Correll, 2010). In the above example, one might expect vaccinated individuals to prioritize a heart attack patient over an unvaccinated person with severe COVID-19.

Allocation preferences may also be driven by perceived prosociality. Vaccinated individuals typically perceive those who are not vaccinated against infectious diseases as antisocial and favor penalties for this free-riding behavior (Korn, Böhm, Meier, \& Betsch, 2020). On this view, vaccinated patients would be prioritized, irrespective of diagnosis. If laypeople assign different priorities on the basis of vaccination status, one question that arises is whether they would apply these criteria for patients with differing prognoses. For instance, if an unvaccinated patient with COVID-19 is more likely to benefit from intensive care than a vaccinated heart attack patient (perhaps because he is younger), the principle of maximum benefit implies that the COVID-19 patient should be prioritized. However, on the grounds of reciprocity and responsibility discussed above, some laypeople might disagree, especially if they are themselves vaccinated.

As public support is vital for the successful implementation of any approach to resource allocation (Simonsen \& Robbins, 2018), we conducted a survey-based experiment to examine the German public's preferences regarding admission to intensive care. In a series of scenarios, participants were asked repeatedly to decide between patients who differed in terms of health condition, expected treatment benefits, and vaccination status. We further investigated whether those decisions varied when patients were to be prioritized for available versus depleted resources. In general, experts agree that it is ethically permissible to reallocate critical care to someone who is more likely to benefit (Biddison et al., 2014; Nates et al., 2016). While there is no medical or moral difference between withholding and withdrawing intensive care, the latter often incurs a higher emotional tax (Beck, van de Loo, \& Reiter-Theil, 2008). Consequently, allocation preferences of the public could be biased against the withdrawal of medical resources too. Our study was designed to identify any such bias among the German public. In exploring lay preferences in the context of medical ethical dilemmas, it was not our intention to criticize or propose any revision of well-founded principles for resource allocation. Instead, we hope the results will inform communication 
strategies to explain and justify triage procedures, thereby increasing public understanding and support.

\section{Method}

\section{Participants and research design}

The experiment was conducted in Germany during the fourth wave of the coronavirus pandemic (November 16-17, 2021) as part of the COVID-19 Snapshot Monitoring (COSMO) cross-sectional study series (Betsch, Wieler, \& Habersaat, 2020). Participants were recruited from a non-probabilistic sample $(N=1,014)$, which was quota-representative for age $\times$ gender and federal state. Participants ranged in age from 18 to 74 years $(M=44.69$, $S D=15.49)$ and included 497 males and 517 females. A large majority $(87 \%, n=878)$ had already been vaccinated against COVID-19. Participants were randomly assigned to one of two experimental conditions: equal versus unequal likelihood of benefit from intensive care for patients with COVID-19 and heart failure.

\section{Procedure and materials}

After assessing the relevant information about demographics and vaccination status, participants were asked to complete a series of allocation decisions in two distinct scenarios and to evaluate the extent of different patients' own responsibility for requiring intensive care.

\section{Scenario 1: Allocation of intensive care}

In the first scenario, all participants were told that many intensive care units were reaching their capacity limit because of the high incidence of coronavirus cases. They were asked to imagine that two patients needed the only available ICU bed, which meant that intensive care would be withheld from one of them. Participants in the equal likelihood of benefit group were told that the two patients were similar, that their chances of survival without intensive care were comparably low, and that both would benefit equally from an ICU bed. In the unequal likelihood of benefit group, participants were told that the two patients were similar but that their chances of survival without intensive treatment might differ. They were further asked to imagine that intensive care was expected to stabilize all patients equally well- that is, all would have the same probability of survival with intensive treatment.

After reading the relevant information, participants in both groups were asked to make a series of six consecutive decisions prioritizing one of two patients for intensive care. In total, there were four patients competing against each other: a vaccinated and unvaccinated patient with COVID-19 and a vaccinated and unvaccinated heart attack patient. In the equal 
likelihood of benefit condition, participants were told only the patients' diagnosis and vaccination status. In the unequal likelihood of benefit group, participants were also told that the COVID-19 patients had a $25 \%$ probability of survival without intensive care while the heart attack patients had a $50 \%$ chance. In each case, the order of presentation of decisions and patients was randomized.

\section{Scenario 2: Reallocation of intensive care}

In the second scenario, participants were asked to imagine that all beds in an ICU were occupied by unvaccinated patients with COVID-19 and that a vaccinated heart attack patient had shown up. As in scenario 1, participants in the equal likelihood of benefit condition were told that the chances of survival without intensive care were comparably low for all patients and that all patients would benefit equally from being admitted to the ICU. Accordingly, participants in the unequal likelihood of benefit condition were asked to imagine that the COVID-19 patients had a $25 \%$ probability of survival without intensive care while the heart attack patients had a 50\% chance. Again, they were asked to imagine that all patients would have the same probability of survival if admitted to the ICU, indicating a higher likelihood of benefit for the COVID-19 patients. After reading the relevant information, all participants were asked to decide whether the vaccinated heart attack patient should be admitted to intensive care, which would mean reallocating resources withdrawn from one of the unvaccinated COVID-19 patients. Again, the order of the two options (admitting versus not admitting the heart attack patient) was randomized.

\section{Blaming patients}

Participants were also asked to assess the extent of four patients' responsibility for their poor state of health and their need for admission to the ICU. As in scenario 1, the question related to a vaccinated and unvaccinated patient with COVID-19 and a vaccinated and unvaccinated heart attack patient. Participants were asked to respond on Likert-type scales ranging from 1 (not to blame at all) to 5 (very much to blame).

\section{Results}

\section{Allocation decisions}

A loglinear Bradley-Terry model (Hatzinger \& Dittrich, 2012) was used to estimate average patient priority from the six pairwise comparisons for vaccinated and unvaccinated participants in the two experimental conditions in scenario 1. Figure 1 shows how patients were prioritized - equally, higher, or lower than the baseline of the vaccinated patient with COVID-19 (second row). On average, vaccinated participants in the equal likelihood of benefit condition (left side, black) prioritized vaccinated heart attack patients (first row) 
above this baseline. Unvaccinated patients with heart attack (third row) and vaccinated patients with COVID-19 were similarly prioritized, and unvaccinated patients with COVID19 (fourth row) were deprioritized as compared to all other patients. In the unequal likelihood of benefit condition, vaccinated participants (left side, red) assigned lower priority to heart attack patients; that is, heart attack patients who were less likely to benefit from ICU treatment were prioritized at the same level as COVID-19 patients. In this case, priority depended on vaccination status alone, with vaccinated patients prioritized over unvaccinated patients.

Unvaccinated participants responded differently. In the equal likelihood of benefit condition (right side, black), heart attack patients and unvaccinated patients with COVID-19 were prioritized over vaccinated patients with COVID-19. In the unequal likelihood of benefit condition (right side, red), the assigned priorities differed by vaccination status rather than diagnosis, as unvaccinated patients were prioritized over vaccinated individuals.

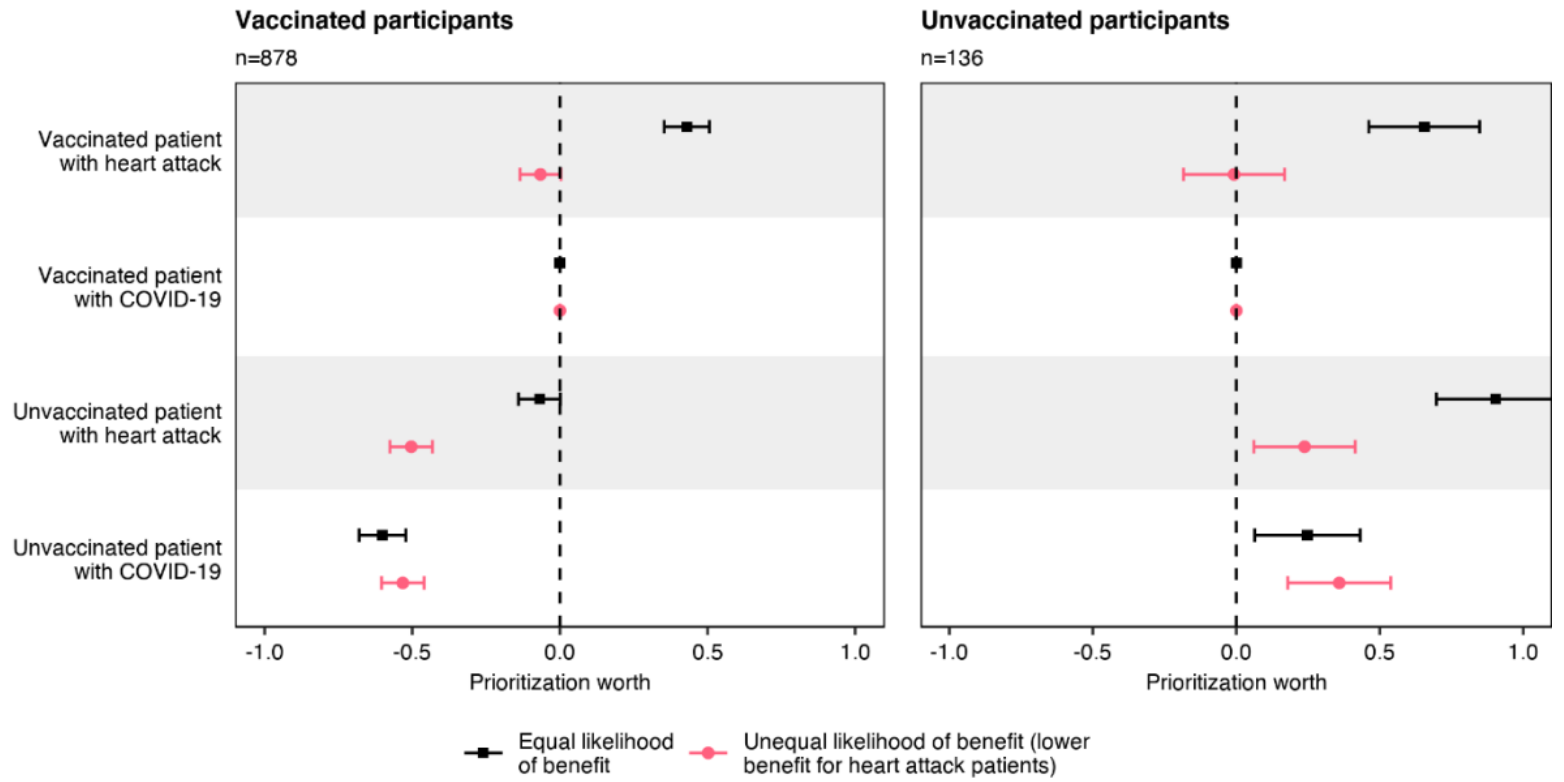

\section{Figure 1. Prioritization for intensive care.}

Note: The Bradley-Terry model estimates the prioritization of each of the four patients from a series of six pairwise comparisons. Log transformation was applied to compare prioritization of each group against a baseline (vaccinated patient with COVID-19). Positive values indicate higher priority relative to the baseline, and negative values indicate lower priority. Error bars indicate 95\% confidence intervals (CIs). Non-overlapping CIs indicate that prioritizations differ by participant vaccination status and experimental manipulation. In the equal likelihood of benefit condition, participants were told that COVID-19 and heart attack patients were similar; that is, their chances of survival without intensive care were comparably low, and both would benefit equally from intensive care. In the unequal likelihood of benefit group, participants were told that the chances of survival without intensive treatment were higher for the heart attack patients (50\%) than for the COVID-19 patients $(25 \%)$ and that the latter would benefit more from intensive care. According to established allocation principles (Emanuel et al., 2020), COVID-19 patients should be prioritized over heart attack patients in the unequal likelihood of benefit condition, regardless of their vaccination status. However, this pattern was not confirmed; instead, vaccinated participants prioritized vaccinated patients, indicating the influence of perceived reciprocity and responsibility. 


\section{Withholding versus withdrawing intensive care}

Figure 2 shows how participants decided when intensive care should be allocated to a vaccinated heart attack patient or to an unvaccinated COVID-19 patient. A majority of vaccinated participants (left) prioritized the vaccinated heart attack patient over the unvaccinated patient with COVID-19, regardless of experimental condition or scenario. For unvaccinated participants (right), the vaccinated heart attack patient was assigned abovechance treatment priority in the equal likelihood of benefit condition when care was to be withheld (scenario 1) and below-chance probability in the unequal likelihood of benefit condition when care was to be withdrawn (scenario 2).

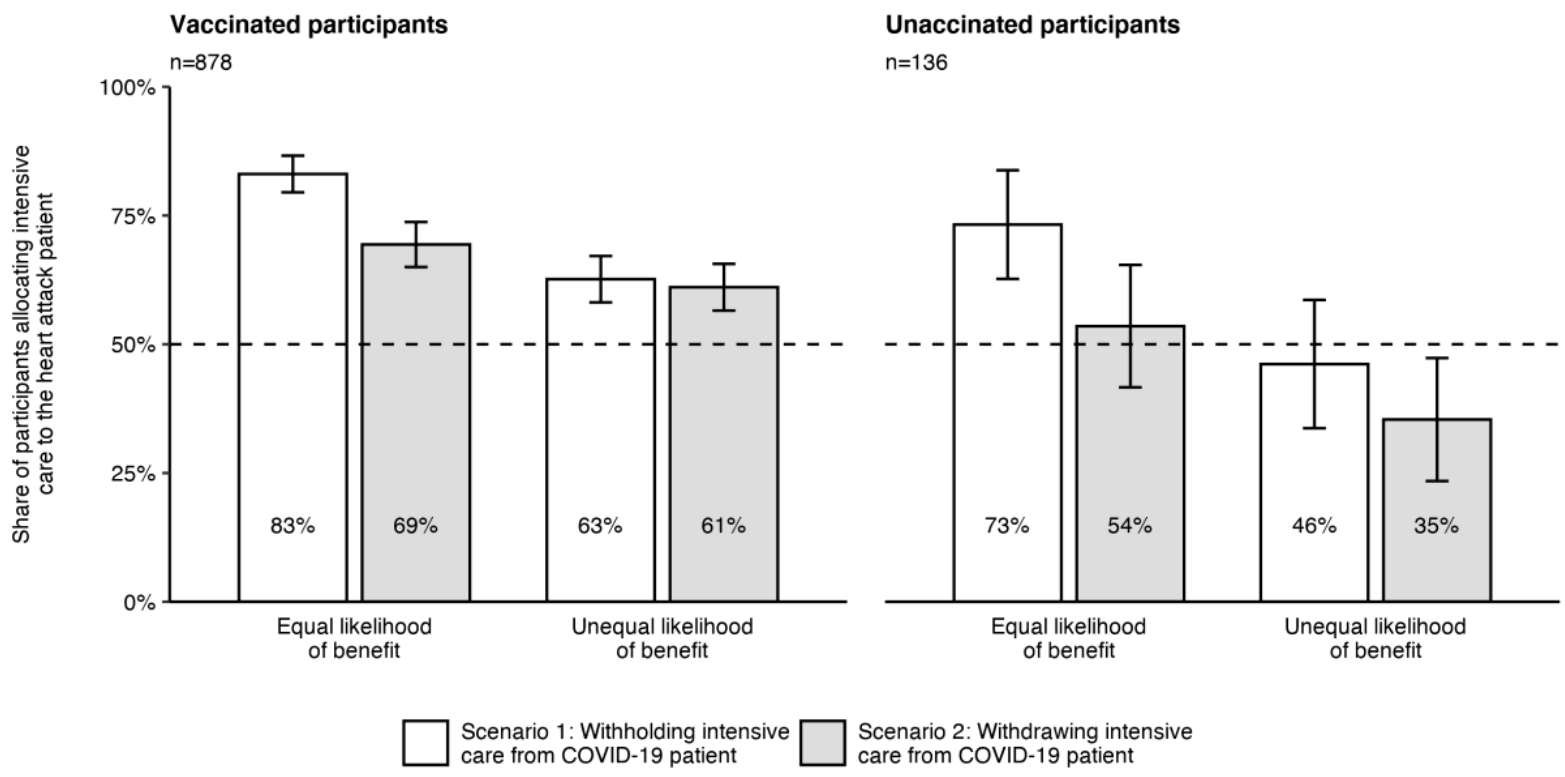

Figure 2. Prioritization of a vaccinated heart attack patient over an unvaccinated patient with COVID-19.

Note: Vaccinated participants (left panel) and unvaccinated participants (right panel) were asked to decide whether they would allocate intensive care to a vaccinated heart attack patient or an unvaccinated patient with COVID-19. The likelihood of benefit varied across participants; in the unequal likelihood condition, the COVID-19 patient had a higher likelihood of benefit. All participants made allocation decisions in two scenarios. In scenario 1, treating the heart attack patient meant withholding intensive care from the COVID-19 patient; in scenario 2, treating the heart attack patient meant withdrawing intensive care from the COVID-19 patient. Allocation decisions differed by participant vaccination status, patient likelihood of benefit, and whether intensive care was to be withhold or withdrawn. On average, vaccinated participants decided to treat the vaccinated heart attack patient even if this patient was less likely to benefit from intensive care. Error bars visualize $95 \%$ confidence intervals.

A mixed effects logistic regression was performed to examine differences across participants, experimental conditions, and scenarios. As shown in Table 1, the probability of treating a vaccinated heart attack patient in preference to an unvaccinated patient with COVID-19 increased when participants were vaccinated, when both patients had an equal 
likelihood of benefit, and when treating the vaccinated heart attack patient meant withholding rather than withdrawing intensive care from an unvaccinated patient with COVID-19.

Table 1. Predictors of prioritization of a vaccinated heart attack patient over an unvaccinated COVID-19 patient.

\begin{tabular}{lcc}
\hline Predictor & OR & 95\% CI \\
\hline Constant & 0.54 & $0.37-0.80$ \\
Between subject factors & & \\
$\quad$ Vaccinated (Baseline: Unvaccinated) & 2.66 & $1.81-3.92$ \\
$\quad$ Equal likelihood of benefit (Baseline: Unequal likelihood) & 2.54 & $1.93-3.36$ \\
Within subject factor & & \\
$\quad$ Withholding treatment (Baseline: Withdrawing treatment) & 1.69 & $1.36-2.11$ \\
\hline
\end{tabular}

Note: Odds ratios (OR) and 95\% confidence intervals (CI) from mixed effects logistic regression (ICC $=.31$, marginal $R^{2}=.08$, conditional $R^{2}=.36$ ). All predictors were significant with $p<.01$. An extended model checking for interaction effects between predictors revealed no such interactions (see online supplement).

\section{Blaming patients for their poor health}

Figure 3 shows average blame ratings for the four patient types by diagnosis and vaccination status. Pairwise comparisons of vaccinated and unvaccinated participants' ratings revealed significant differences regarding unvaccinated patients with COVID-19, $t(183.57)=$ $16.80, p<.001$, and heart attack, $t(205.09)=4.54, p<.001$. While both vaccinated and unvaccinated participants tended not to blame vaccinated patients for their poor health, vaccinated participants were more likely to blame unvaccinated patients, especially those with COVID-19. 


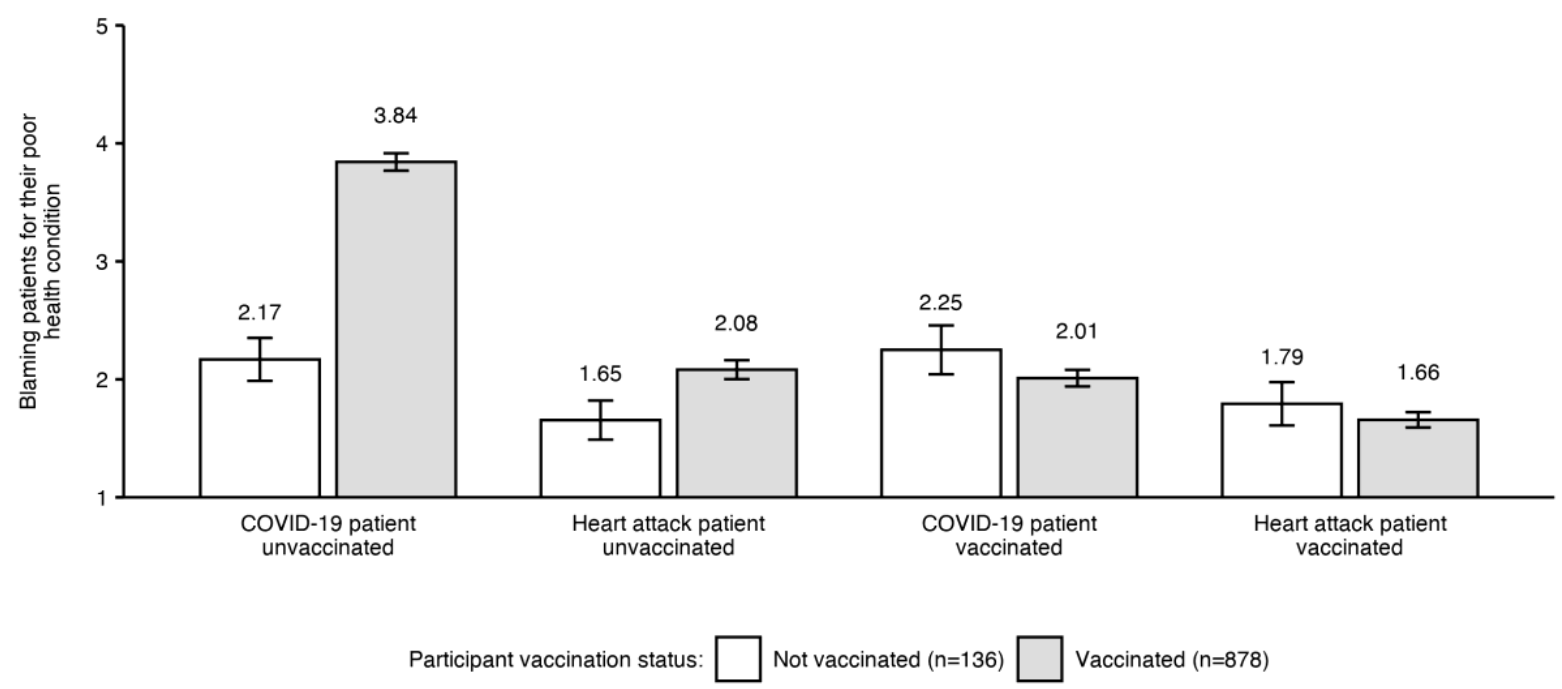

Figure 3. Blame of patients for their poor health.

Note: Participants were asked to what extent patients with different diagnoses and vaccination status were to blame for their own poor health and need for intensive care on scales ranging from 1 (not to blame at all) to 5 (very much to blame). In most cases, blame ratings were low. However, vaccinated participants strongly agreed that unvaccinated patients with COVID-19 were to blame for their poor health. This attitude may explain the deprioritization of unvaccinated COVID-19 patients in the allocation decision tasks. Bar labels indicate means, and error bars visualize $95 \%$ confidence intervals.

\section{Discussion}

When medical resources are scarce, there is a need to define and implement allocation procedures. In the case of intensive care, experts agree that allocation should be based on the principle of maximizing benefits (Emanuel et al., 2020; Vincent \& Creteur, 2020), prioritizing patients who would benefit more from ICU admission. This approach is ethically permissible even if it means that intensive care must be withdrawn from another patient (Biddison et al., 2014; Nates et al., 2016). However, when two patients with comparable likelihood of benefit compete for intensive care, random selection is often recommended (Persad et al., 2009). While other factors like instrumental value may be taken into account, ethicists agree that patient wealth or social status should not inform decision making (Vincent $\&$ Creteur, 2020). Assigning blame is also considered unacceptable; that is, patients who are responsible for their own disease must not be deprioritized (Hansson, 2018). It follows that patients should be treated equally regardless of their vaccination status (Emanuel et al., 2020). When an unvaccinated patient with COVID-19 and a vaccinated heart attack patient are equally likely to benefit from intensive care, both should have the same chance for treatment.

However, our data indicate that laypeople may adopt other perspectives, depending on their own vaccination status. Vaccinated participants disagreed with the prevailing 
professional view by deprioritizing unvaccinated patients with COVID-19 and blaming them for their poor health. Non-vaccination was seen as violating a social contract (Korn et al., 2020), and deprioritization can be understood as an act of punishment. Interestingly, when equal likelihood of benefit was assumed, similar mid-level priority was assigned to the unvaccinated heart attack patient and the vaccinated patient with COVID-19 while the vaccinated heart attack patient was assigned superior priority. Consequently, both vaccination status and diagnosis influenced allocation preference, with heart attack prioritized over COVID-19.

When COVID-19 patients are expected to benefit more than heart attack patients from intensive care, they should be prioritized. In this case, however, participants' decisions took no account of the diagnosis, confirming discrimination against COVID-19 patients across conditions. A possible explanation is that vaccinated people may prefer to maintain normal ICU services (i.e., for conditions other than COVID-19), distributing only remaining capacity (if any) to those with COVID-19. In this regard, heart attack is a common cause of ICU admission in non-pandemic times and represents perhaps the central case of emergency admission.

In contrast, unvaccinated participants tended not to blame patients for their health status. However, their prioritization preferences were not driven by benefit considerations alone, as unvaccinated patients with COVID-19 or heart attack were prioritized over vaccinated patients with COVID-19 in both the equal and unequal likelihood of benefit conditions. Overall, the results indicate an ingroup bias (Scheepers, Spears, Doosje, \& Manstead, 2006), as both vaccinated and unvaccinated participants tended to allocate resources according to their own status beyond established allocation principles.

On average, participants preferred withholding rather than withdrawing treatment. While this approach diverges from ethical considerations (Biddison et al., 2014; Nates et al., 2016), it aligns with previous evidence regarding the high emotional burden experienced by healthcare workers in withdrawing resources from patients in need (Beck et al., 2008). Laypeople are also likely to experience this burden, which would explain the preference for treating a vaccinated heart attack patient when this meant withholding (rather than withdrawing) ICU resources from an unvaccinated COVID-19 patient.

While our results highlight important differences between lay decision making and medical ethics regarding intensive care allocation, we must acknowledge some limitations of the present study. First, the allocation decisions were fictitious, and preferences may differ when real lives are at stake. Second, our findings relate to a specific time and context: a 
German sample surveyed at a time when infection rates and ICU admissions from the delta variant were rising quickly. Future research should investigate prioritization preferences in other countries and cultural contexts, as well as timelines (i.e., during versus after the current pandemic). Third, our results may have been biased because responsibility indicators were provided for COVID-19 patients but not for heart attack patients. Future research should explore whether lay preferences diverge when taking account of patients' vaccination status and other health-related behaviors (e.g., diet, exercise). Fourth, the allocation tasks forced participants to prioritize one patient over another, but research from other domains shows that people often prefer not to participate in clinical decision making (Levinson, Kao, Kuby, \& Thisted, 2005). As there was no option to refrain from allocation or to assign equal priorities, participants who had no preference were obliged to select one patient at random, which means that some answers may not perfectly reflect individual preferences. However, as rankings were analyzed across participants, the results can be considered a reliable representation of allocation preferences among German adults aged less than 75 years.

The discrepancy between allocation recommendations based on a set of established ethical principles and lay preferences suggests some potential for social conflict. To mitigate any such tensions, it seems important to address the underlying problem of low vaccination rates that leads to overburdened ICUs. This can be achieved by (1) communication strategies that persuade unvaccinated people to get vaccinated (e.g., by informing them about the severe effects of infection and potential or actual overburdening of ICUs) and (2) policies that limit the contacts of unvaccinated people or mandate vaccination. However, when new virus strains emerge and the available vaccines prove less effective, the burden on ICUs may remain high even when large parts of the population are vaccinated; in this regard, the recent discovery of the Omicron variant warrants special attention (Karim \& Karim, 2021). More generally, procedures and principles for allocating intensive care need to be well documented and clearly communicated to enhance public understanding through large-scale education campaigns. Clearly, the allocation process should also be explained to patients and their relatives when seeking intensive care.

\section{Online supplement}

Materials, data, and the data analysis script are available at https://osf.io/twbzu/?view only=784bf9836c93498890b28c4d7c38e819 [link will be replaced by DOI upon publication].

\section{Ethical declaration}


Our research obtained ethical clearance from the University of Erfurt's IRB (\#20200302/20200501) and all participants provided informed consent prior to data collection.

\section{Author contributions}

PS, LK, LF, SE and CB designed the research; PS and LK performed the research; PS planned and performed data analysis; PS wrote the initial draft, which was revised and approved by all authors.

\section{Funding}

This work was supported by German Research Foundation (BE3970/11-1, BE3970/12-1), Federal Centre for Health Education, Robert Koch Institute, Leibniz Institute of Psychology, Klaus Tschira Stiftung, Thüringer Ministerium für Wirtschaft, Wissenschaft und digitale Gesellschaft, Thüringer Staatskanzlei and University of Erfurt (no award/grant numbers).

\section{Acknowledgments}

The study was conducted as part of Germany's COVID-19 Snapshot Monitoring (COSMO), a joint project of the University of Erfurt, the Robert Koch Institute, the Federal Centre for Health Education, the Leibniz Institute of Psychology, the Science Media Center, the Bernhard Nocht Institute for Tropical Medicine, and the Yale Institute for Global Health. We are grateful to Dr. Philipp Felgendreff for the fruitful discussions that inspired this research.

\section{References}

Beck, S., van de Loo, A., \& Reiter-Theil, S. (2008). A "little bit illegal”? Withholding and withdrawing of mechanical ventilation in the eyes of German intensive care physicians. Medicine, Health Care and Philosophy, 11(1), 7-16. https://doi.org/10.1007/s11019007-9097-8

Betsch, C., Wieler, L. H., \& Habersaat, K. (2020). Monitoring behavioural insights related to COVID-19. The Lancet, 395(10232), 1255-1256. https://doi.org/10.1016/S01406736(20)30729-7

Biddison, L. D., Berkowitz, K. A., Courtney, B., De Jong, C. M. J., Devereaux, A. V., Kissoon, N., ... Powell, T. (2014). Ethical Considerations. Chest, 146(4), e145S-e155S. https://doi.org/10.1378/chest.14-0742

Burki, T. K. (2021). Lifting of COVID-19 restrictions in the UK and the Delta variant. The Lancet Respiratory Medicine, 9(8), e85. https://doi.org/10.1016/S2213-2600(21)00328-3

Christian, M. D., Hawryluck, L., Wax, R. S., Cook, T., Lazar, N. M., Herridge, M. S., ... Burkle, F. M. (2006). Development of a triage protocol for critical care during an influenza pandemic. Canadian Medical Association Journal, 175(11), 1377-1381. 
https://doi.org/10.1503/cmaj.060911

Decety, J., Echols, S., \& Correll, J. (2010). The Blame Game: The Effect of Responsibility and Social Stigma on Empathy for Pain. Journal of Cognitive Neuroscience, 22(5), 985997. https://doi.org/10.1162/jocn.2009.21266

del Rio, C., Malani, P. N., \& Omer, S. B. (2021). Confronting the Delta Variant of SARSCoV-2, Summer 2021. JAMA, 326(11), 1001. https://doi.org/10.1001/jama.2021.14811

Emanuel, E. J., Persad, G., Upshur, R., Thome, B., Parker, M., Glickman, A., ... Phillips, J. P. (2020). Fair Allocation of Scarce Medical Resources in the Time of Covid-19. New England Journal of Medicine, 382(21), 2049-2055. https://doi.org/10.1056/NEJMsb2005114

Hansson, S. O. (2018). The Ethics of Making Patients Responsible. Cambridge Quarterly of Healthcare Ethics, 27(1), 87-92. https://doi.org/10.1017/S0963180117000421

Hatzinger, R., \& Dittrich, R. (2012). prefmod : An R Package for Modeling Preferences Based on Paired Comparisons, Rankings, or Ratings. Journal of Statistical Software, 48(10). https://doi.org/10.18637/jss.v048.i10

Karim, S. S. A., \& Karim, Q. A. (2021). Omicron SARS-CoV-2 variant: a new chapter in the COVID-19 pandemic. The Lancet, 398(10317), 2126-2128. https://doi.org/10.1016/S0140-6736(21)02758-6

Korn, L., Böhm, R., Meier, N. W., \& Betsch, C. (2020). Vaccination as a social contract. Proceedings of the National Academy of Sciences, 117(26), 14890-14899. https://doi.org/10.1073/pnas.1919666117

Levinson, W., Kao, A., Kuby, A., \& Thisted, R. A. (2005). Not all patients want to participate in decision making. Journal of General Internal Medicine, 20(6), 531-535. https://doi.org/10.1111/j.1525-1497.2005.04101.x

Marckmann, G., Neitzke, G., Schildmann, J., Michalsen, A., Dutzmann, J., Hartog, C., ... Janssens, U. (2020). Entscheidungen über die Zuteilung intensivmedizinischer Ressourcen im Kontext der COVID-19-Pandemie. Medizinische Klinik - Intensivmedizin Und Notfallmedizin, 115(6), 477-485. https://doi.org/10.1007/s00063-020-00708-w

Nacoti, M., Ciocca, A., Giupponi, A., Brambillasca, P., Lussana, F., Pisano, M., ... Montaguti, C. (2020). At the Epicenter of the Covid-19 Pandemic and Humanitarian Crises in Italy: Changing Perspectives on Preparation and Mitigation. NEJM Catalyst. https://doi.org/10.1056/CAT.20.0080

Nates, J. L., Nunnally, M., Kleinpell, R., Blosser, S., Goldner, J., Birriel, B., ... Sprung, C. L. (2016). ICU Admission, Discharge, and Triage Guidelines. Critical Care Medicine, 
44(8), 1553-1602. https://doi.org/10.1097/CCM.0000000000001856

Our World in Data. (2021). Coronavirus (COVID-19) Vaccinations. Retrieved December 1, 2021, from https://ourworldindata.org/covid-vaccinations

Persad, G., Peek, M. E., \& Emanuel, E. J. (2020). Fairly Prioritizing Groups for Access to COVID-19 Vaccines. JAMA, 324(16), 1601. https://doi.org/10.1001/jama.2020.18513

Persad, G., Wertheimer, A., \& Emanuel, E. J. (2009). Principles for allocation of scarce medical interventions. The Lancet, 373(9661), 423-431. https://doi.org/10.1016/S01406736(09)60137-9

Scheepers, D., Spears, R., Doosje, B., \& Manstead, A. S. R. (2006). The social functions of ingroup bias: Creating, confirming, or changing social reality. European Review of Social Psychology, 17(1), 359-396. https://doi.org/10.1080/10463280601088773

Simonsen, W., \& Robbins, M. D. (2018). Citizen Participation in Resource Allocation. Routledge. https://doi.org/10.4324/9780429501630

Tagesschau. (2021). Wir werden zu kämpfen haben. Retrieved December 1, 2021, from https://www.tagesschau.de/inland/gesellschaft/bayern-corona-hochinzidenzgebiet101.html

Tenforde, M. W., Olson, S. M., Self, W. H., Talbot, H. K., Lindsell, C. J., Steingrub, J. S., ... Zunie, T. (2021). Effectiveness of Pfizer-BioNTech and Moderna Vaccines Against COVID-19 Among Hospitalized Adults Aged $\geq 65$ Years — United States, JanuaryMarch 2021. MMWR. Morbidity and Mortality Weekly Report, 70(18), 674-679. https://doi.org/10.15585/mmwr.mm7018e1

Vincent, J.-L., \& Creteur, J. (2020). Ethical aspects of the COVID-19 crisis: How to deal with an overwhelming shortage of acute beds. European Heart Journal: Acute Cardiovascular Care, 9(3), 248-252. https://doi.org/10.1177/2048872620922788

Wichmann, O., Scholz, S., Waize, M., Schmid-Küpke, N., Hamouda, O., Wieler, L. H., \& Schaade, L. (2021). Welche Impfquote ist notwendig, um COVID-19 zu kontrollieren? Epidemiologisches Bulletin, (27), 3-13. https://doi.org/10.25646/8742 